\title{
Nasal and extra-nasal methicillin resistant Staphylococcus aureus colonization among hemodialysis patients; is routine culturing of other body sites necessary?
}

\author{
Narges-Sadat Zahed ${ }^{1 \oplus}$, Zohreh Aminzadeh $^{2 *}{ }^{\star}$, Atefeh Sadat Akhavi Mirab ${ }^{3}$, Latif Gachkar² ${ }^{2}$ Ali Akhbari ${ }^{4}$ \\ ${ }^{1}$ Department of Nephrology, Shahid Beheshti University of Medical Sciences, Tehran, Iran \\ ${ }^{2}$ Infectious Disease and Tropical Medicine Research Center, Shahid Beheshti University of Medical Sciences, Tehran, Iran \\ ${ }^{3}$ Pediatric Infections Research Center, Research Institute for Children Health, Shahid Beheshti University of Medical Sciences, Tehran, Iran \\ ${ }^{4}$ Ashrafi Esfahani Hospital, Shahid Beheshti University of Medical Sciences, Tehran, Iran
}

\section{A R T I C L E I N F O}

Article Type:

Original

\section{Article History:}

Received: 29 December 2017

Accepted: 18 April 2018

Published online: 20 May 2018

\section{Keywords:}

Hemodialysis

Staphylococcal infections

Nasal colonization

Extra-nasal carrier

\begin{abstract}
A B S T R A C T
Introduction: Asymptomatic Staphylococcus aureus carriers have become a great concern because of being at risk of subsequent $S$. aureus infections. The role of nasal $S$. aureus carriages as an endogenous source for staphylococcal infections especially methicillin resistant S. aureus (MRSA) infections has been known that seems to be contributed to morbidity, mortality, and also the cost of end-stage renal disease management. Besides, many recent studies have demonstrated that extranasal sites may be important unrecognized reservoirs for resistant $S$. aureus.

Objectives: The purpose of the present research was to identify the frequency and factors associated with extra-nasal S. aureus colonization among maintenance hemodialysis patients.

Patients and Methods: A total of 179 hemodialysis patients were enrolled in this study. Swab cultures were obtained from anterior nares, posterior pharynx, and the inguinal area. Culture plates were analyzed for the presence of methicillin-resistant or methicillin-susceptible S. aureus using standard microbiological techniques for $S$. aureus and MRSA.

Results: 113 out of 179 patients (63\%) were men and $66(37 \%)$ were women with the mean age of $59.8 \pm 13.6$ years. 36 out of 179 patients (20\%) were colonized with $S$. aureus which 5 patients (2.7\%) were colonized with MRSA. Prevalence of extra-nasal S. aureus colonization was $12 \%$ ( $22 / 179$ patients), the prevalence of nasal S. aureus colonization was $10 \%$ (18/179 patients) and $2.7 \%$ of patients (5/179 patients) were colonized with S. aureus in more than one body site. Around 3 out of 5 MRSA colonized patients $(60 \%)$ were extra-nasal carriers. There was a significant association between type of venous access for dialysis with the extra- nasal colonization $(P=0.03)$ and also an association between underlying disease of diabetes mellitus type 2 with the extra-nasal colonization $(P=0.01)$.

Conclusion: This study has emphasized the importance of extra-nasal evaluation along with nasal site sampling as an endogenous risk factor for staphylococcal infections among hemodialysis patients.
\end{abstract}

Implication for health policy/practice/research/medical education:

In a study on 179 hemodialysis patients, we found the importance of extra-nasal evaluation along with nasal site sampling as an endogenous risk factor for staphylococcal infections among hemodialysis patients.

Please cite this paper as: Zahed NS, Aminzadeh Z, Akhavi Mirab AS, Gachkar L, Akhbari A. Nasal and extra-nasal methicillin resistant Staphylococcus aureus colonization among hemodialysis patients; is routine culturing of other body sites necessary? J Renal Inj Prev. 2018;7(4):280-285. DOI: 10.15171/jrip.2018.62.

\section{Introduction}

The risk of healthcare-associated infections, in particular those caused by Staphylococcus aureus including methicillinresistant S. aureus (MRSA) are high (1). S. aureus has been isolated as one of the most common pathogens causing bloodstream infections among hemodialysis patients (2).
The risk of invasive S. aureus and MRSA infection among patients receiving maintenance hemodialysis is higher than the general population. Although the rate of annual invasive MRSA infections in the general population has been reported at 0.2-0.4 infections per 1000 persons (3), rates among patients receiving maintenance hemodialysis 
have been estimated at 37 cases per 1000 persons (4) that shows a 100 fold higher risk. The mortality rate following an invasive MRSA infection among patients on maintenance hemodialysis accounts for $17 \%$ (4).

Asymptomatic $S$. aureus carriers have become a great concern because of being at risk of subsequent $S$. aureus infections (5-7). The role of nasal S. aureus carriages as an endogenous source for staphylococcal infections especially MRSA infections has been known that seems to be contributed to morbidity, mortality, and also the cost of end-stage renal disease management (2). Besides, many recent studies have demonstrated that extra-nasal sites may be important unrecognized reservoirs for MRSA (8-15).

MRSA colonization in the nares, axilla, inguinal area, and rectum of admitted patients with an $S$. aureus infection was $25,6,11$, and $13 \%$, respectively and overall $37 \%$ of them were colonized by MRSA (16). A study of households with a history of a recent $S$. aureus skin infection demonstrated that up to $50 \%$ of household members were colonized by $S$. aureus and a nares-only survey would miss $48 \%$ of S. aureus colonization and $51 \%$ of MRSA colonization (17). Nasal screening alone, compared with multiple-site screening, missed approximately $27 \%$ of MRSA colonized patients at hospital admission in a French study (15). Because body colonization, especially of extra-nasal sites, is more widespread than initially believed, interventions to prevent $S$. aureus and MRSA infection may need to consider extra-nasal decolonization as well as traditional nasal decolonization with agents such as mupirocin (18).

\section{Objectives}

The purpose of the present research was to identify the frequency and factors associated with extra-nasal S. aureus colonization among maintenance hemodialysis patients.

\section{Patient and Methods}

Study population

This cross-sectional investigation conducted at four hemodialysis centers in Tehran, Iran from March to June 2017. All adults receiving dialysis at participating centers who had inclusion criteria were eligible for participation. Inclusion criteria were; being on maintenance hemodialysis, and not using any kind of antibiotics since two weeks before sampling. The patients gave their written informed consent prior to sampling.

Separate sterile-cotton-tripped swabs were rotated into the patients' anterior nares and pharynx as well as using sterile swabs soaked in trypticase soy broth (TSB) to be rubbed on the skin of the groins. The specimens were cultured in $5 \%$ sheep blood agar and incubated at $37^{\circ} \mathrm{C}$ for 24 hours. S. aureus isolates were identified based on their colony morphologies, biochemical activities and the coagulase test (19). Susceptibility patterns to different antibiotics, including cefoxitin $(30 \mu \mathrm{g})$, penicillin (10 IU), clindamycin $(2 \mu \mathrm{g})$, erythromycin $(15 \mu \mathrm{g})$, vancomycin $(30$ $\mu \mathrm{g})$, cotrimoxazole $(1.25 / 23.75 \mu \mathrm{g})$, doxycycline $(30 \mu \mathrm{g})$, and rifampin $(5 \mu \mathrm{g})$ were determined with the guidelines of the Clinical and Laboratory Standards Institute (CLSI), using the Kirby-Bauer disk diffusion method (20). Methicillin resistance susceptibility was determined by using cefoxitin $(30 \mu \mathrm{g})$ disks (PadtanTeb, Iran) using Muller-Hinton agar plates inoculated with a suspension (equivalent to $0.5 \mathrm{McF}$ arland standards) of the $S$. aureus clinical isolates. The plates were incubated at $35^{\circ} \mathrm{C}$ for 24 hours and inhibition zones were measured $(21,22)$. The CLSI 2010 criteria were used for interpretation. Cefoxitin disk diffusion tests regarding $S$. aureus $\geq 20 \mathrm{~mm}$ was considered as susceptible and $\leq 19 \mathrm{~mm}$ as resistant (23). The double disk approximation test (D-test) was applied for evaluating the inducible clindamycin resistance (ICR). Therefore, erythromycin $(15 \mu \mathrm{g})$ and clindamycin $(2 \mu \mathrm{g})$ disks were set in close proximity $(15-20 \mathrm{~mm})$ on an agar plate inoculated with a standardized suspension of the isolate. Plates were analyzed after 24 hours incubation at $37^{\circ} \mathrm{C}(24)$. Since the zone of inhibition around the clindamycin disk on the side facing the erythromycin disk is flattened ( $\mathrm{D}$ shaped), the isolate was classified as having ICR (positive D-test) (25).

A questioner form was completed for each patient including the past medical history and behavioral risk factors for $S$. aureus colonization.

\section{Ethical issues}

1) The research followed the tenets of the Declaration of Helsinki. 2) Informed consent was obtained 3). This study was approved by the Ethics Committee of Shahid Beheshti University of Medical Sciences. This work has been extracted from the thesis of Atefeh Sadat Akhavi Mirab in School of Medicine, Shahid Beheshti University of Medical Sciences (\# 81m).

\section{Statistical analysis}

Data were analyzed by using the SPSS version 16 . We used descriptive statistics to describe the basic features of the data and to determine association between variables. $P$ value $<0.05$ was considered statistically significant.

Results

A total of 179 patients were enrolled with the mean age of $59.8 \pm 13.6$ years (a range of 22 to 87 years). 113 (63\%) patients were men and $66(37 \%)$ were women. Table 1 shows the baseline characteristic of the patients. Table 2 indicates the distribution of hemodialysis patients based on the body site of $S$. aureus colonization. Overall, 36 out of 179 patients (20\%) were colonized with S. aureus which 5 patients $(2.7 \%)$ were colonized with MRSA. Prevalence of extra-nasal S. aureus colonization was $12 \%(22 / 179$ patients), the prevalence of nasal $S$. aureus colonization was $10 \%(18 / 179$ patients) and $2.7 \%$ of patients $(5 / 179$ patients) were colonized with $S$. aureus in more than one body site. The prevalence of MRSA colonization in nares, inguinal, and pharynx were $1.7 \%(3 / 179), 1.2 \%(2 / 179)$ 
Table 1. Baseline characteristic associated with extra nasal S. aureus colonization among hemodialysis patients, Tehran, Iran, MarchJune 2017

\begin{tabular}{|c|c|c|c|c|c|c|}
\hline & $\begin{array}{l}\text { All patients } \\
(n=179)\end{array}$ & $\begin{array}{c}\text { Extra-nasal } \\
\text { colonization }(n=22)\end{array}$ & $\begin{array}{c}\text { No extra-nasal } \\
\text { colonization }(n=157)\end{array}$ & OR & $95 \% \mathrm{Cl}$ & $P$ value \\
\hline Age (y) Mean+SD & $59.8 \pm 13.9$ & $58.5 \pm 11.95$ & $59.98 \pm 14.18$ & - & - & - \\
\hline Male gender & $113(63.12 \%)$ & $15(68.2 \%)$ & $98(62.42 \%)$ & 1.3 & $0.497,3.34$ & 0.64 \\
\hline History of a skin or soft-tissue infection & $6(3.35 \%)$ & $1(4.5 \%)$ & $5(3.18 \%)$ & 1.45 & $0.16,12.9$ & 0.55 \\
\hline Hospitalization in the previous 3 months & $50(27.93 \%)$ & $5(22.7 \%)$ & $45(28.6 \%)$ & 0.73 & $0.25,2.1$ & 0.56 \\
\hline History of surgery in the previous 3 months & $20(11.17 \%)$ & $1(4.5 \%)$ & $19(12 \%)$ & 0.34 & $0.04,27$ & 0.47 \\
\hline History of antibiotics in the previous 3 months & $54(30.16 \%)$ & $5(22.7 \%)$ & $49(31.2 \%)$ & 0.65 & $0.22,1.85$ & 0.41 \\
\hline \multicolumn{7}{|l|}{ Type of vascular access } \\
\hline Venous catheter & $60(34.5 \%)$ & $3(13.6 \%)$ & $58(36.9 \%)$ & & & \\
\hline Arteriovenous graft or fistula & $119(66.5 \%)$ & $19(86 \%)$ & $99(63 \%)$ & 0.27 & $0.07,0.95$ & \\
\hline Duration of dialysis more than 1 year & $146(81.6 \%)$ & $19(86 \%)$ & $127(80.8 \%)$ & 0.67 & $0.18,2.4$ & 0.77 \\
\hline Diabetic mellitus & $71(39.7 \%)$ & $14(63.6 \%)$ & $57(36.3 \%)$ & 3.07 & $1.2,7.7$ & 0.01 \\
\hline Coronary disease & $94(52.5 \%)$ & $14(63.6 \%)$ & $80(50.9 \%)$ & 1.68 & $0.67,4.2$ & 0.26 \\
\hline Polycystic disease & $7(3.9 \%)$ & $1(4.5 \%)$ & $6(3.8 \%)$ & 1.12 & $0.13,10.4$ & 1 \\
\hline
\end{tabular}

and $0.5 \%(1 / 179)$ respectively. Three out of five MRSA colonized patients $(60 \%)$ were extra-nasal carriers.

There was a significant association between type of venous access for dialysis with the extra-nasal colonization (95\% CI: $0.07-0.95, P=0.03)$ and also an association between underlying disease of diabetes mellitus type 2 with the extra-nasal colonization (95\% CI: 1.2-7.7, $P=0.01$ ). There were associations between extra-nasal $S$. aureus colonization with a dialysis length of more than one year and also with a history of skin and soft tissue infections even though these associations were not statistically significant.

Four out of 18 patients with nasal colonization (22\%) had been detected to be colonized by $S$. aureus in their extra-nasal sites, whereas 18 out of 161(11\%) non-nasal colonized patients were colonized by $S$. aureus in their extra-nasal sites.

All MRSA isolates were susceptible to vancomycin and rifampin. The resistant pattern of MSSA and MRSA isolates has shown in Tables 3 and 4 respectively.

\section{Discussion}

This study showed a $20 \%$ prevalence of S. aureus colonization following a survey on their anterior nares, pharynx and inguinal regions with $2.7 \%$ of MRSA colonization that is very lower than the study by Eells et al with a prevalence of $42 \%$ and $6 \%$ for S. aureus and MRSA (26). This difference might be related to the techniques. In this survey, anterior nares of patients were the site of $S$. aureus colonization in $10 \%$ (18 patients) which is less than other research findings that reported at 33\%, 46\% and $43 \%$ (27-29). This might be explained by the number of enrolled patients in their studies. Extra-nasal colonization prevalence in our research was $12 \%$ which is very lower than the study of Eells et al (26) that could be due to their higher colonization rates overall.

Four out of 18 patients (22\%) in the present study with nasal S. aureus colonization also had extra-nasal colonization but the results of the study by Baker and et al showed that $56 \%$ patients with nasal S. aureus colonization had extranasal colonization (30). This difference may be due to obtaining samples from more sites of body (oropharynx, axilla, hand, perirectal, wound, and catheter insertion site) in their study in comparison with our research that was done from inguinal region and oropharynx as extra nasal carrier investigations.

Eighteen out of 161(11\%) non-nasal colonized patients in our study were colonized by S. aureus in their extranasal sites which means extra-nasal surveillance resulted in $11 \%$ additionally identification of $S$. aureus carriers. This finding is less than the results of other studies which reported up to $33 \%$ additional detection $(26,31)$. However, all reports have emphasized the benefit of extra-nasal investigations in addition to nasal surveys.

The prevalence of MRSA colonization in the present study was $2.7 \%$ which is similar to $\mathrm{Lu}$ et al with a prevalence of $2.36 \%$ (32) and less than $5.6 \%-12 \%$ reported by others $(26,33,34)$. The MRSA isolates showed a sensitivity of a $100 \%$ to vancomycin and rifampin, $50 \%$ to clindamycin and erythromycin and $83 \%$ to cotrimoxazole and doxycycline. In the study by Mohajeri et al (35), sensitivity to cotrimoxazole and doxycycline was $29 \%$ and $50 \%$ respectively and in the study of Tashakori et al was 25\% (36). In the study of Wu et al susceptibility to vancomycin, tigecycline, rifampin and clindamycin was $100 \%, 100 \%$, $95.7 \%, 2.1 \%$ respectively (37).

We found a significant association between types of venous access for dialysis with the extra-nasal colonization which is different with the result of Eells et al. We also determined a significant association between underlying disease of diabetes mellitus type 2 with the extra-nasal colonization which is similar to the study by Lederer et al (33). In the study of Lederer et al, S. aureus nasal colonization had a relationship with old aging, diabetes mellitus and previous hospitalization (33). Our research indicated a non-significant association between extra- 
Table 2. The distribution of hemodialysis patients based on the body site of S. aureus colonization $(n=179)$

\begin{tabular}{|c|c|}
\hline & $\begin{array}{c}\text { Percentage of colonized } \\
\text { patients, No. (\%) }\end{array}$ \\
\hline \multicolumn{2}{|c|}{ Anybody site colonization } \\
\hline S. aureus & $36(20)$ \\
\hline MSSA & $31(86)$ \\
\hline MRSA & $5(14)$ \\
\hline \multicolumn{2}{|c|}{ Nasal colonization } \\
\hline S. aureus & $18(10)$ \\
\hline MSSA & $15(83)$ \\
\hline MRSA & $3(17)$ \\
\hline \multicolumn{2}{|c|}{ Throat colonization } \\
\hline S. aureus & $15(8)$ \\
\hline MSSA & $14(93.4)$ \\
\hline MRSA & $1(6.6)$ \\
\hline \multicolumn{2}{|c|}{ Inguinal-region colonization } \\
\hline S. aureus & $8(4.5)$ \\
\hline MSSA & $6(75)$ \\
\hline MRSA & $2(25)$ \\
\hline \multicolumn{2}{|c|}{ Extra-nasal colonization } \\
\hline S. aureus & $22(12)$ \\
\hline MSSA & $19(86.3)$ \\
\hline MRSA & $3(13.7)$ \\
\hline \multicolumn{2}{|c|}{ Colonization at $>1$ body site } \\
\hline S. aureus & $5(2.7)$ \\
\hline MSSA & $4(80)$ \\
\hline MRSA & $1(20)$ \\
\hline \multicolumn{2}{|c|}{ Extra-nasal colonization only } \\
\hline S. aureus & $18(10)$ \\
\hline MSSA & $15(83.3)$ \\
\hline MRSA & $3(6.7)$ \\
\hline \multicolumn{2}{|c|}{ Nasal colonization only } \\
\hline S. aureus & $14(7.8)$ \\
\hline MSSA & $12(85.7)$ \\
\hline MRSA & $2(14.2)$ \\
\hline \multicolumn{2}{|c|}{ Throat colonization only } \\
\hline S. aureus & $12(6.7)$ \\
\hline MSSA & $11(91.6)$ \\
\hline MRSA & $1(8.3)$ \\
\hline \multicolumn{2}{|c|}{ Inguinal-region colonization only } \\
\hline S. aureus & $5(2.7)$ \\
\hline MSSA & $4(80)$ \\
\hline MRSA & $1(20)$ \\
\hline
\end{tabular}

nasal S. aureus colonization with a history of skin and soft tissue infections. The findings of Eells et al are similar to our results (26).

\section{Conclusion}

In summary, this study has emphasized that extra-nasal investigations for $S$. aureus detect additional $S$. aureus carries and also detect more MRSA colonized hemodialysis people. Moreover, extra-nasal investigations might be helpful to recognize the original site of staphylococcal infections in cases of recurrent infections. Besides, it might be a strategy to decrease colonization pressure if necessary.

Limitations of the study

In this research, we did not evaluate all possible body sites for $S$. aureus colonization affecting the number of carriage. Besides, the sample size of our study was not ideal due to our limited resources which could have increased the chance of missing colonized people.

\section{Acknowledgments}

This work has been extracted from the thesis of Atefeh Sadat Akhavi Mirab (\# 81m). The authors would like to thank the vice chancellor for research and technology for financial support and clinical Research Development Center of Loghman Hakim Hospital,Shahid Beheshti University of Medical Science for its help.

\section{Authors' contribution}

NSZ; study design and manuscript drafting. ZA; study design, manuscript reviewing. ASAM; data collection and statistical analysis. LG; statistical analysis. AA; do laboratory work.

\section{Conflicts of interest}

The authors declare no conflict of interest.

\section{Ethical considerations}

Ethical issues (including plagiarism, data fabrication, double publication) have been completely observed by the authors.

Table 3. Resistant pattern of MSSA, hemodialysis patients, Tehran, Iran, March-June 2017 ( $n=35)$

\begin{tabular}{lccccccc}
\hline \multirow{2}{*}{$\begin{array}{l}\text { Susceptibility } \\
\text { results }\end{array}$} & \multicolumn{7}{c}{ Antibiotics } \\
\cline { 2 - 7 } & Clindamycin & Penicillin & Vancomycin & Doxycycline & Erythromycin & Cotrimoxazole & Rifampin \\
\hline Resistance & $8(23 \%)$ & $11(31.5 \%)$ & $4(11.5 \%)$ & $7(20 \%)$ & $15(43 \%)$ & $6(17 \%)$ & $3(8.5 \%)$ \\
Intermediate & - & - & - & - & $1(3 \%)$ & - & $29(8)$ \\
Sensitive & $27(77 \%)$ & $24(68.5 \%)$ & $31(88.5 \%)$ & $28(80 \%)$ & $19(54 \%)$ & $32(91.5 \%)$ \\
\hline
\end{tabular}

Table 4. Resistant pattern of MRSA, hemodialysis patients, Tehran, Iran, March-June 2017 ( $n=6)$

\begin{tabular}{|c|c|c|c|c|c|c|c|}
\hline \multirow{2}{*}{$\begin{array}{l}\text { Susceptibility } \\
\text { results }\end{array}$} & \multicolumn{7}{|c|}{ Antibiotics } \\
\hline & Clindamycin & Rifampin & Cotrimoxazole & Erythromycin & Doxycycline & Vancomycin & Penicillin \\
\hline Resistance & $3(50 \%)$ & _- & $1(17 \%)$ & $3(50 \%)$ & $1(17 \%)$ & _ & $2(33 \%)$ \\
\hline Intermediate & - & - & - & - & - & - & - \\
\hline Sensitive & $3(50 \%)$ & $6(100 \%)$ & $5(83 \%)$ & $3(50 \%)$ & $5(83 \%)$ & $6(100 \%)$ & $4(67 \%)$ \\
\hline
\end{tabular}




\section{Funding/Support}

This project was funded by Pediatric Infectious Disease Research Center of Mofid hospital, Shahid Beheshti University of Medical.

\section{Reference}

1. Lowy FD. Staphylococcus aureus infections. N Engl J Med. 1998;339:520-32. doi: 10.1056/NEJM199808203390806.

2. Patel PR, Kallen AJ, Arduino MJ. Epidemiology, surveillance, and prevention of bloodstream infections in hemodialysis patients. Am J Kidney Dis. 2010;56:566-77. doi: $\quad 10.1053 /$ j.ajkd.2010.02.352.

3. Fridkin SK, Hageman JC, Morrison M, Sanza LT, Como-Sabetti K, Jernigan JA, et al. Methicillin-resistant Staphylococcus aureus disease in three communities. N Engl J Med. 2005;352:1436-44. doi: 10.1056/NEJMoa043252.

4. Nguyen DB, Lessa FC, Belflower R, Mu Y, Wise M, Nadle $\mathrm{J}$, et al. Invasive methicillin-resistant Staphylococcus aureus infections among patients on chronic dialysis in the United States, 2005-2011. Clin Infect Dis. 2013;57:1393-400. doi: $10.1093 / \mathrm{cid} / \mathrm{cit} 546$.

5. Casewell MW. The nose: an underestimated source of Staphylococcus aureus causing wound infection. J Hosp Infect. 1998;40:S3-11.

6. Von Eiff C, Becker K, Machka K, Stammer H, Peters G. Nasal carriage as a source of Staphylococcus aureus bacteremia. Study Group. N Engl J Med. 2001;344:11-6. doi: 10.1056/NEJM200101043440102.

7. Wertheim HF, Vos MC, Ott A, van Belkum A, Voss A, Kluytmans JA, et al. Risk and outcome of nosocomial Staphylococcus aureus bacteraemia in nasal carriers versus non-carriers. Lancet. 2004;364:703-5.

8. Chang S, Sethi AK, Eckstein BC, Stiefel U, Cadnum JL, Donskey CJ. Skin and environmental contamination with methicillin-resistant Staphylococcus aureus among carriers identified clinically versus through active surveillance. Clin Infect Dis. 2009;48:1423-8. doi: 10.1086/598505.

9. Mody L, Kauffman CA, Donabedian S, Zervos M, Bradley SF. Epidemiology of Staphylococcus aureus colonization in nursing home residents. Clin Infect Dis. 2008;46:1368-73. doi: $10.1086 / 586751$.

10. Rohr U, Wilhelm M, Muhr G, Gatermann S. Qualitative and (semi)quantitative characterization of nasal and skin methicillin-resistant Staphylococcus aureus carriage of hospitalized patients. Int J Hyg Environ Health. 2004:207:51-5. doi: 10.1078/1438-4639-00266.

11. Ringberg $\mathrm{H}$, CathrinePetersson $\mathrm{A}$, Walder $\mathrm{M}$, Hugo Johansson PJ. The throat: an important site for MRSA colonization. Scand J Infect Dis. 2006;38:888-93. doi: 10.1080/00365540600740546.

12. Kerttula AM, Lyytikäinen O, Virolainen A, Finne-Soveri H, Agthe N, Vuopio-Varkila J. Staphylococcus aureus colonization among nursing home residents in a large Finnish nursing home. Scand J Infect Dis. 2007;39:9961001. doi: 10.1080/00365540701466207.

13. Roghmann MC, Gorman PH, Wallin MT, Kreisel K, Shurland S, Johnson JA. Staphylococcus aureus colonization in community-dwelling people with spinal cord dysfunction. Arch Phys Med Rehabil. 2007;88:979-83. doi: 10.1016/j.apmr.2007.05.005.

14. Aizen E, Ljubuncic Z, Ljubuncic P, Aizen I, Potasman I. Risk factors for methicillin-resistant Staphylococcus aureus colonization in a geriatric rehabilitation hospital. J Gerontol A Biol Sci Med Sci. 2007;62:1152-6.

15. Eveillard M, de Lassence A, Lancien E, Barnaud G, Ricard JD, Joly-Guillou ML. Evaluation of a strategy of screening multiple anatomical sites for methicillin-resistant Staphylococcus aureus at admission to a teaching hospital. Infect Control Hosp Epidemiol. 2006;27:181-4.

16. Yang ES, Tan J, Eells S, Rieg G, Tagudar G, Miller LG. Body site colonization in patients with community-associated methicillin-resistant Staphylococcus aureus and other types of S. aureus skin infections. Clin Microbiol Infect. 2010;16:425-31. doi: 10.1111/j.1469-0691.2009.02836.x.

17. Miller LG, Eells SJ, Taylor AR, David MZ, Ortiz N, Zychowski D, et al. Staphylococcus aureus colonization among household contacts of patients with skin infections: risk factors, strain discordance, and complex ecology. Clin Infect Dis. 2012;54:1523-35. doi: 10.1093/cid/cis213.

18. Kallen AJ, Jernigan JA, Patel PR. Decolonization to prevent infections with Staphylococcus aureus in patients undergoing hemodialysis: a review of current evidence. Semin Dial. 2011;24:533-9. doi: 10.1111/j.1525-139X.2011.00959.x.

19. Forbes BE, Sahm DF, Weissfeld AS, Trevino EA. Bailey \&Scotts Diagnostic Microbiology 11th ed. The C.V. Mosby Company, Toronto, 2002

20. Clinical and Laboratory Standards Institute/CLSI (2005) Performance standards for antimicrobial susceptibility for antimicrobial susceptibility testing; Fifteen Informational Supplement. CLSI/NCCLS documents M100-S15 .USA

21. Skov R, Smyth R, Clausen M, Larsen AR, Frimodt-Møller $\mathrm{N}$, Olsson-Liljequist B, et al. Evaluation of a cefoxitin 30 microg disc on Iso-Sensitest agar for detection of methicillin-resistant Staphylococcus aureus. J Antimicrob Chemother. 2003;52:204-7.

22. Velasco D, del Mar Tomas M, Cartelle M, Beceiro A, Perez A, Molina F, et al. Evaluation of different methods for detecting methicillin (oxacillin) resistance in Staphylococcus aureus . J Antimicrob Chemother. 2005;55:379-82.

23. Wayne P A. Clinical and Laboratory Standards Institute/ NCCLS, Performance Standards for Antimicrobial Susceptibility Testing. Fifteenth Informational Supplement, 2014, CLSI/NCCLS document M100-S15. CLSI

24. Sedighi I, Moez HJ, Alikhani MY. Nasal carriage of methicillin resistant Staphylococcus aureus and their antibiotic susceptibility patterns in children attending daycare centers. Acta Microbiol Immunol Hung. 2011;58:22734. doi: 10.1556/AMicr.58.2011.3.6.

25. Saxena AK, Panhotra BR, Chopra R. Advancing age and the risk of nasal carriage of Staphylococcus aureus among patients on long-term hospital-based hemodialysis. Ann Saudi Med. 2004;24:337-42.

26. Eells SJ, Kalantar-Zadeh K, Bolaris MA, May L, Miller LG. Body site Staphylococcus aureus colonization among maintenance hemodialysis patients. Nephron. 2015;129:7983. doi: $10.1159 / 000369348$.

27. Johnson LB, Jose J, Yousif F, Pawlak J, Saravolatz LD. Prevalence of colonization with community-associated methicillin-resistant Staphylococcus aureus among endstage renal disease patients and healthcare workers. Infect Control Hosp Epidemiol. 2009;30:4-8. doi: 10.1086/592983.

28. Aminzadeh Z, Mastari Farahani A, Gchkar L. Prevalence of Staphylococcus aureus carriage in patients on hemodialysis and the pattern of antibacterial resistance. Iran J Clin Infect 
Dis. 2006;1:5-10.

29. Köseoğlu O1, Sayın Kutlu S, Cevahir N. Prevalence and risk factors for methicillin-resistant Staphylococcus aureus colonization among outpatients undergoing hemodialysis treatment. Mikrobiyol Bul. 2012;46:106-12.

30. Baker SE, Brecher SM, Robillard E, Strymish J, Lawler E, Gupta K. Extranasal methicillin-resistant Staphylococcus aureus colonization at admission to an acute care Veterans Affairs hospital. Infect Control Hosp Epidemiol. 2010;31:42-6. doi: 10.1086/649222.

31. McKinnell JA, Huang SS, Eells SJ, Cui E, Miller LG. Quantifying the impact of extranasal testing of body sites for methicillin-resistant Staphylococcus aureus colonization at the time of hospital or intensive care unit admission. Infect Control Hosp Epidemiol. 2013;34:161-70. doi: 10.1086/669095.

32. Lu PL, Tsai JC, Chiu YW, Chang FY, Chen YW, Hsiao $\mathrm{CF}$, et al. Methicillin-resistant Staphylococcus aureus carriage, infection and transmission in dialysis patients, healthcare workers and their family members. Nephrol Dial Transplant. 2008;23:1659-65. doi: 10.1093/ndt/gfm806.

33. Lederer SR, Riedelsdorf G, Schiffl H. Nasal carriage of meticillin resistant Staphylococcus aureus: the prevalence, patients at risk and the effect of elimination on outcomes among outclinic haemodialysis patients. Eur J Med Res. 2007;12:284-8

34. Hadley AC, Karchmer TB, Russell GB, McBride DG, Freedman BI. The prevalence of resistant bacterial colonization in chronic hemodialysis patients. Am J Nephrol. 2007;27:352-9. doi: 10.1159/000103383.

35. Mohajeri P, Izadi B, Rezaei M, Fallahi B, Moradi Z, Zare ME. Study of nasal carriage Methicillin resistant Staphylococcus aureus in hemodialysis patients in Kermanshah. Behbood. 2012;15:485-91.

36. Tashakori M, Mohseni Moghadam F, Ziasheikholeslami N, Jafarpour P, Behsoun M, Hadavi M, et al. Staphylococcus aureus nasal carriage and patterns of antibiotic resistance in bacterial isolates from patients and staff in a dialysis center of southeast Iran. Iran J Microbiol. 2014;6:79-83.

37. Wu HS, Kuo SC, Chen LY, Chiang MC, Lin YT, Wang FD, et al. Comparison between patients under hemodialysis with community-onset bacteremia caused by communityassociated and healthcare-associated methicillin-resistant Staphylococcus aureus strains. J Microbiol Immunol Infect. 2013;46:96-103. doi: 10.1016/j.jmii.2012.02.004.

Copyright @ 2018 The Author(s); Published by Nickan Research Institute. This is an open-access article distributed under the terms of the Creative Commons Attribution License (http://creativecommons.org/licenses/by/4.0), which permits unrestricted use, distribution, and reproduction in any medium, provided the original work is properly cited. 